2002

\title{
Upper bound for the non-maximal eigenvalues of irreducible nonnegative matrices
}

Xiao-Dong Zhang

Rong Luo

Follow this and additional works at: https://researchrepository.wvu.edu/faculty_publications

\section{Digital Commons Citation}

Zhang, Xiao-Dong and Luo, Rong, "Upper bound for the non-maximal eigenvalues of irreducible nonnegative matrices" (2002).

Faculty Scholarship. 424.

https://researchrepository.wvu.edu/faculty_publications/424

This Article is brought to you for free and open access by The Research Repository @ WVU. It has been accepted for inclusion in Faculty Scholarship by an authorized administrator of The Research Repository @ WVU. For more information, please contact ian.harmon@mail.wvu.edu. 


\section{Czechoslovak Mathematical Journal}

Xiao-Dong Zhang; Rong Luo

Upper bound for the non-maximal eigenvalues of irreducible nonnegative matrices

Czechoslovak Mathematical Journal, Vol. 52 (2002), No. 3, 537-544

Persistent URL: http://dml.cz/dmlcz/127741

\section{Terms of use:}

(C) Institute of Mathematics AS CR, 2002

Institute of Mathematics of the Czech Academy of Sciences provides access to digitized documents strictly for personal use. Each copy of any part of this document must contain these Terms of use.

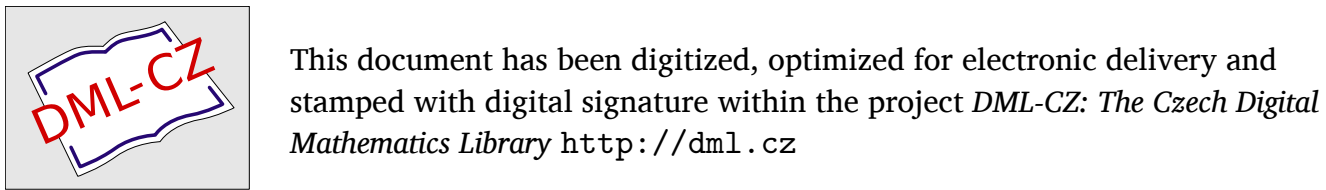




\title{
UPPER BOUND FOR THE NON-MAXIMAL EIGENVALUES OF IRREDUCIBLE NONNEGATIVE MATRICES
}

\author{
XiaO-Dong Zhang, Hefei, and Rong Luo, Morgantown
}

(Received July 27, 1999)

\begin{abstract}
We present a lower and an upper bound for the second smallest eigenvalue of Laplacian matrices in terms of the averaged minimal cut of weighted graphs. This is used to obtain an upper bound for the real parts of the non-maximal eigenvalues of irreducible nonnegative matrices. The result can be applied to Markov chains.
\end{abstract}

Keywords: eigenvalue, irreducible nonnegative matrix, averaged minimal cut

MSC 2000: 15A42, 05C50

\section{INTRODUCTION}

The matrices in this paper are real and square. The eigenvalues of an $n \times n$ matrix $A$ are arranged in the non-increasing order with respect to their real parts:

$$
\operatorname{Re} \lambda_{1}(A) \geqslant \operatorname{Re} \lambda_{2}(A) \geqslant \ldots \geqslant \operatorname{Re} \lambda_{n}(A)
$$

Given $n$ real numbers $a_{1}, a_{2}, \ldots, a_{n}$, denote by $\bar{a}=\max \left\{a_{i}: 1 \leqslant i \leqslant n\right\}$ and $\underline{a}=$ $\min \left\{a_{i}: 1 \leqslant i \leqslant n\right\}$.

For a given $n \times n$ symmetric nonnegative matrix $C=\left(c_{i j}\right)$, we associate a weighted graph $G_{c}=(V, E)$ with $V=\{1,2, \ldots, n\},(i, j) \in E$ if and only if $c_{i j}>0$ and $i \neq j$, and the weight of the edge $(i, j)$ is $c_{i j}$. Let $r_{i}$ be the $i$-th row sum of $C, i=1,2, \ldots, n$. Then

$$
L\left(G_{c}\right)=\operatorname{diag}\left(r_{1}, r_{2}, \ldots, r_{n}\right)-C
$$

Supported by National Natural Science Foundation of China under Grant 19671077 and the Doctoral Program Foundation of Higher Education of China. 
is the Laplacian matrix of the weighted graph $G_{c}$ [5] (if $C$ is a $(0,1)$-matrix, then $L\left(G_{c}\right)=L(G)$ is the Laplacian matrix of $\left.G\right)$. It is easily seen that $L\left(G_{c}\right)$ is a singular, positive semidefinite matrix. Moreover, if $C$ is irreducible, then $\lambda_{n-1}\left(L\left(G_{c}\right)\right)>$ $\lambda_{n}\left(L\left(G_{c}\right)=0\right.$.

Let $G_{c}$ be a weighted graph. The edge-density [6], [7] of a subset $M$ of the vertex set $V$ is defined to be

$$
\varrho_{c}(M)=\sum_{i \in M, j \notin M} \frac{c_{i j}}{|M|(n-|M|)},
$$

and, the averaged minimal cut $[2],[6]$ of $G_{c}$ is defined to be

$$
\gamma\left(G_{c}\right)=\min \left\{\varrho_{c}(M): 0<|M|<n\right\}
$$

where $|M|$ is the cardinality of the set $M$. Since $\gamma\left(G_{c}\right)=0$ if and only if $C$ is reducible, it is also called the averaged measure [2] of irreducibility of $C$.

In Section 2 we use $\gamma\left(G_{c}\right)$ to obtain a lower and an upper bound for $\lambda_{n-1}\left(L\left(G_{c}\right)\right)$ (i.e., the algebraic connectivity of $G_{c}$ [5], [6]). This, in turn, will be applied to obtain, in Section 3, an upper bound for real parts of the non-maximal eigenvalues of irreducible nonnegative matrices. This has applications to Markov chains in Section 4.

\section{LAPLACIAN MATRICES}

In order to prove our results, we first give the following inequality which may be of independent interest.

Lemma 2.1. If $n$ positive numbers $d_{1}, d_{2}, \ldots, d_{n}$ and $n$ real numbers $x_{1}$, $x_{2}, \ldots, x_{n}$ satisfy the condition $\sum_{i=1}^{n} x_{i} / d_{i}=0$, then

$$
\sum_{i=1}^{n-1} i(n-i)\left(x_{i}-x_{i+1}\right)^{2} \geqslant 2 \underline{d} \sum_{i=1}^{n} \frac{x_{i}^{2}}{d_{i}} .
$$

Proof. Let the $n \times n$ matrix $S=\left(s_{i j}\right)$ correspond to the quadratic form of the left-hand side in (5). It is easily seen that $S$ is a symmetric positive semidefinite matrix with the eigenvectors $e=(1,1, \ldots, 1)^{T}$ and $f=(n-1, n-3, n-5, \ldots,-n+1)^{T}$ corresponding to the eigenvalues $\lambda_{n}(S)=0$ and $\lambda_{n-1}(S)=2$, respectively (cf. [2]). Thus $S-2 I_{n}$ has only one negative eigenvalue, where $I_{n}$ is the identity matrix. 
Denote $D=\operatorname{diag}\left(d_{1}, d_{2}, \ldots, d_{n}\right)$. Since $D^{\frac{1}{2}}\left(S-2 I_{n}\right) D^{\frac{1}{2}}$ is congruent to $S-2 I_{n}$, $D^{\frac{1}{2}}\left(S-2 I_{n}\right) D^{\frac{1}{2}}$ and $S-2 I_{n}$ have the same numbers of positive, negative and zero eigenvalues. Therefore $\lambda_{n-1}\left(D^{\frac{1}{2}}\left(S-2 I_{n}\right) D^{\frac{1}{2}}\right)=0$. Thus by [4, p. 242],

$$
0 \leqslant \lambda_{n-1}\left(D^{\frac{1}{2}} S D^{\frac{1}{2}}\right)+\lambda_{1}(-2 D)=\lambda_{n-1}\left(D^{\frac{1}{2}} S D^{\frac{1}{2}}\right)-2 \underline{d} .
$$

Hence, by the Courant-Fischer Theorem and in view of the identity $\sum_{i=1}^{n} x_{i} / d_{i}=0$, we have that,

$$
\begin{aligned}
2 \underline{d} \leqslant \lambda_{n-1}\left(D^{\frac{1}{2}} S D^{\frac{1}{2}}\right) & =\min _{y^{T} D^{-\frac{1}{2}} e=0} \frac{y^{T} D^{\frac{1}{2}} S D^{\frac{1}{2}} y}{y^{T} y} \\
& =\min _{z^{T} D^{-1} e=0} \frac{z^{T} S z}{z^{T} D^{-1} z} \leqslant \frac{x^{T} S x}{x^{T} D^{-1} x},
\end{aligned}
$$

where $z=D^{\frac{1}{2}} y$ and $x=\left(x_{1}, x_{2}, \ldots, x_{n}\right)^{T}$. Therefore (5) holds.

Theorem 2.2. Let $G_{c}$ be a weighted connected graph (i.e., $C=\left(c_{i j}\right)$ is irreducible) with $n$ vertices. Let $D=\operatorname{diag}\left(d_{1}, d_{2}, \ldots, d_{n}\right)$ be a positive diagonal matrix and $\Omega=D L\left(G_{c}\right)$. Then

$$
2 \underline{d} \gamma\left(G_{c}\right) \leqslant \lambda_{n-1}(\Omega) \leqslant n \bar{d} \gamma\left(G_{c}\right) .
$$

Proof. Since $\Omega$ is similar to $D^{\frac{1}{2}} L\left(G_{c}\right) D^{\frac{1}{2}}$, all of the eigenvalues of $\Omega$ are real and $\lambda_{n}(\Omega)=0$. Let $x=\left(x_{1}, x_{2}, \ldots, x_{n}\right)^{T}$ be the real eigenvector of $\Omega$ corresponding to the eigenvalue $\lambda_{n-1}(\Omega)$, i.e.,

$$
D L\left(G_{c}\right) x=\lambda_{n-1}(\Omega) x .
$$

Without loss of generality, we assume that $x_{1} \geqslant x_{2} \geqslant \ldots \geqslant x_{n}$ and $L\left(G_{c}\right)=\left(l_{i j}\right)$. So $\sum_{j=1}^{n} l_{i j}=\sum_{i=1}^{n} l_{i j}=0$. Hence by $(7)$,

$$
\begin{aligned}
\sum_{i=1}^{m} \lambda_{n-1}(\Omega) \frac{x_{i}}{d_{i}} & =\sum_{i=1}^{m} \sum_{j=1}^{n} l_{i j} x_{j}=\sum_{i=1}^{m} \sum_{j=1}^{m} l_{i j} x_{j}+\sum_{i=1}^{m} \sum_{j=m+1}^{n} l_{i j} x_{j} \\
& =\sum_{j=1}^{m}\left(-\sum_{i=m+1}^{n} l_{i j}\right) x_{j}+\sum_{i=1}^{m} \sum_{j=m+1}^{n} l_{i j} x_{j} \\
& =\sum_{j=1}^{m} \sum_{i=m+1}^{n}\left(-l_{i j} x_{j}\right)+\sum_{i=1}^{m} \sum_{j=m+1}^{n} l_{i j} x_{j} \\
& =\sum_{i=1}^{m} \sum_{j=m+1}^{n}\left(-l_{i j}\left(x_{i}-x_{j}\right)\right) \geqslant \sum_{i=1}^{m} \sum_{j=m+1}^{n}-l_{i j}\left(x_{m}-x_{m+1}\right) \\
& \geqslant \gamma\left(G_{c}\right) m(n-m)\left(x_{m}-x_{m+1}\right) .
\end{aligned}
$$


Multiplying the above inequality by $x_{m}-x_{m+1}$ and summing them for $m=$ $1,2, \ldots, n-1$, we have

$$
\lambda_{n-1}(\Omega) \sum_{i=1}^{n} \frac{x_{i}^{2}}{d_{i}} \geqslant \gamma\left(G_{c}\right) \sum_{i=1}^{n-1} i(n-i)\left(x_{i}-x_{i+1}\right)^{2},
$$

since $\lambda_{n-1}(\Omega) \sum_{i=1}^{n} \frac{x_{i}}{d_{i}}=e^{T} D^{-1} \Omega x=0$ by $(7)$, where $e=(1,1, \ldots, 1)^{T}$. Combining Lemma 2.1 and (8), we obtain the left inequality in (6).

Let $M_{0}$ be a proper subset of the vertex set $V$ such that $\gamma\left(G_{c}\right)=\varrho_{c}\left(M_{0}\right)$. Define an $n$-dimensional vector $y=\left(y_{1}, y_{2}, \ldots, y_{n}\right)^{T}$ where $y_{i}=\frac{a}{\sqrt{d_{i}}}$ if $i \in M_{0}$, and $y_{i}=-\frac{b}{\sqrt{d_{i}}}$ if $i \notin M_{0}$ where $a=\sum_{i \notin M_{0}} \frac{1}{d_{i}}, b=\sum_{i \in M_{0}} 1 / d_{i}$. It is easily seen that $y^{T} D^{-\frac{1}{2}} e=0$. Hence by the Courant-Fischer Theorem,

$$
\begin{aligned}
\lambda_{n-1}(\Omega) & =\lambda_{n-1}\left(D^{\frac{1}{2}} L\left(G_{c}\right) D^{\frac{1}{2}}\right)=\min _{z D^{-\frac{1}{2}} e=0} \frac{z^{T} D^{\frac{1}{2}} L\left(G_{c}\right) D^{\frac{1}{2}} z}{z^{T} z} \leqslant \frac{y^{T} D^{\frac{1}{2}} L\left(G_{c}\right) D^{\frac{1}{2}}}{y^{T} y} \\
& =\gamma\left(G_{c}\right)\left(\frac{1}{a}+\frac{1}{b}\right)\left|M_{0}\right|\left(n-\left|M_{0}\right|\right) \leqslant n \bar{d} \gamma\left(G_{c}\right) .
\end{aligned}
$$

Corollary 2.3 ([2], [7]). Let $G$ be a simple connected graph with $n$ vertices. Then

$$
2 \gamma(G) \leqslant \lambda_{n-1}(L(G)) \leqslant n \gamma(G)
$$

Pro of. It follows from (6) and $d_{1}=d_{2}=\ldots=d_{n}=1$.

Corollary 2.4. Let $G$ be a simple graph with $n$ vertices. Let $A$ be the adjacency matrix and $\Delta, \delta$ be the maximum and the minimum vertex degree of $G$, respectively. Then

$$
\delta-n \gamma(G) \leqslant \lambda_{2}(A) \leqslant \Delta-2 \gamma(G)
$$

Pro of. Since $\delta I_{n}-L(G)=A-\left(\operatorname{diag}\left(r_{1}, r_{2}, \ldots, r_{n}\right)-\delta I_{n}\right)$, we have that $\lambda_{2}\left(\delta I_{n}-L(G)\right) \leqslant \lambda_{2}(A)$. Hence, by (6), the left inequality in (10) holds. In a similar way, the right inequality in (10) is also obtained. 


\section{IRREDUCIBLE NONNEGATIVE MATRICES}

For an $n \times n$ nonnegative matrix $A$ and positive vectors $x$ and $y$ in $\mathbb{R}^{n}$, define

$$
\eta(A, x, y)=\min \frac{\sum_{i \in M, j \notin M}\left(a_{i j} x_{j} y_{i}+a_{j i} x_{i} y_{j}\right)}{2|M|(n-|M|)},
$$

where the minimum is taken over all nonempty subsets $M$ of $\{1,2, \ldots, n\}$. If $y=x$, we denote $\eta(A, x, y)$ by $\eta(A, x)$.

Lemma 3.1. Let $A$ be an $n \times n$ irreducible symmetric nonnegative matrix and let $A u=\lambda_{1}(A) u, u=\left(u_{1}, u_{2}, \ldots, u_{n}\right)>0$. Then

$$
\lambda_{1}(A)-\frac{n}{\underline{u^{2}}} \eta(A, u) \leqslant \lambda_{2}(A) \leqslant \lambda_{1}(A)-\frac{2}{\overline{u^{2}}} \eta(A, u) .
$$

Proof. Let $U=\operatorname{diag}\left(u_{1}, u_{2}, \ldots, u_{n}\right)$ and $C=U A U$. Define $G_{c}$ to be the weighted graph associated with $C$. Then $L\left(G_{c}\right)=U\left(\lambda_{1}(A) I_{n}-A\right) U$. Now choosing $D=U^{-2}$ and $\Omega=U^{-1}\left(\lambda_{1}(A) I_{n}-A\right) U$, it follows that $\lambda_{n-1}(\Omega)=\lambda_{1}(A)-\lambda_{2}(A)$. Since $A$ is symmetric, $A u=\lambda_{1}(A) u, u^{T}=\lambda_{1}(A) u^{T}$ and

$$
\gamma\left(G_{c}\right)=\min \frac{\sum_{i \in M, j \notin M} a_{i j} u_{i} u_{j}}{|M|(n-|M|)}=\eta(A, u) .
$$

Thus, by Theorem 2.2, (12) holds.

Theorem 3.2. Let $A$ be an $n \times n$ irreducible nonnegative matrix. Let $A u=$ $\lambda_{1}(A) u, u>0, v^{T} A=\lambda_{1}(A) v^{T}, v>0, w_{i}=u_{i} v_{i}$. Then

$$
\operatorname{Re} \lambda_{2}(A) \leqslant \lambda_{1}(A)-\frac{2}{\bar{w}} \eta(A, u, v) .
$$

Proof. Let $d_{i}=\sqrt{v_{i} / u_{i}}, D=\operatorname{diag}\left(d_{1}, d_{2}, \ldots, d_{n}\right)$ and $B=\frac{1}{2}\left(D A D^{-1}+\right.$ $\left.\left(D A D^{-1}\right)^{T}\right)$. Then $D^{2} u=v$ and

$$
B(D u)=\frac{D A u+D^{-1} A D^{2} u}{2}=\frac{D \lambda_{1}(A) u+D^{-1} \lambda_{1}(A) v}{2}=\lambda_{1}(A)(D u) .
$$

Moreover, it is easily seen that $\eta(B, D u)=\eta(A, u, v)$ and $(D u)_{i}=\sqrt{u_{i} v_{i}}=\sqrt{w_{i}}$. On the other hand, it follows from [6, p. 237] that $\lambda_{1}(A)+\lambda_{2}(B)=\lambda_{1}(B)+\lambda_{2}(B) \geqslant$ $\lambda_{1}(A)+\operatorname{Re} \lambda_{2}(A)$. Thus (13) follows from (12). 
Corollary 3.3 ([2]). Let $A$ be an $n \times n$ doubly stochastic matrix. Then

$$
\left|1-\lambda_{2}(A)\right| \geqslant 2 \gamma\left(G_{A}\right) .
$$

Pro of. If $A$ is reducible, then $\gamma\left(G_{A}\right)=0$ and (14) holds. We now assume that $A$ is irreducible. Since $A$ is a doubly stochastic matrix, we have that $u=v=$ $(1,1, \ldots, 1)^{T}$ and $\eta(A, u, v)=\gamma\left(G_{A}\right)$. Therefore, by (13),

$$
\left|1-\lambda_{2}(A)\right| \geqslant\left|1-\operatorname{Re} \lambda_{2}(A)\right| \geqslant 2 \gamma\left(G_{A}\right) .
$$

\section{Applications to Markov chains}

Markov chains techniques are often used to model the behavior of large irreducible nearly uncoupled evolutionary systems in which the states naturally divide into $k$-clusters such that the states within each cluster are strongly coupled, but the clusters themselves are only weakly coupled to each other. We may use a stochastic matrix $P$ to describe the states of such a chain. In [3], Hartfiel and Meyer defined the uncoupling measure of $P$ as following:

$$
\sigma(P)=\min \left(\sum_{i \in M_{1}, j \notin M_{1}} p_{i j}+\sum_{i \in M_{2}, j \notin M_{2}} p_{i j}\right),
$$

where the minimum is taken over all nonempty proper subsets $M_{1}, M_{2}$ of $\{1,2, \ldots, n\}$ with $M_{1} \cap M_{2}=\emptyset$.

The following theorem provides the relation between $\sigma(P)$ and $\lambda_{2}(P)$.

Theorem 4.1. Let $P$ be an $n \times n$ irreducible stochastic matrix and $v=$ $\left(v_{1}, v_{2}, \ldots, v_{n}\right)^{T}$ be the stationary distribution vector of $P$. Denote

$$
\mu=\max \left\{\frac{v_{i}}{v_{j}}: 1 \leqslant i, j \leqslant n\right\} .
$$

Then

$$
\sigma(P) \leqslant \frac{2 n^{2}+(-1)^{n}-1}{8} \mu\left|1-\lambda_{2}(P)\right| .
$$


Proof. Since $P$ is a stochastic matrix, we have that $P e=e, e=(1,1, \ldots, 1)^{T}$ and

$$
\begin{aligned}
\eta(P, e, v) & =\min \frac{\sum_{i \in M, j \notin M}\left(p_{i j} v_{i}+p_{j i} v_{j}\right)}{2|M|(n-|M|)} \geqslant \underline{v} \min \frac{\sum_{i \in M, j \notin M}\left(p_{i j}+p_{j i}\right)}{2|M|(n-|M|)} \\
& \geqslant \frac{4 \underline{v}}{2 n^{2}+(-1)^{n}-1} \min \sum_{i \in M, j \notin M}\left(p_{i j}+p_{j i}\right)=\frac{4 \underline{v}}{2 n^{2}+(-1)^{n}-1} \sigma(P) .
\end{aligned}
$$

On the other hand, by Theorem 3.2,

$$
\left|1-\lambda_{2}(P)\right| \geqslant\left|1-\operatorname{Re} \lambda_{2}(P)\right| \geqslant \frac{2 \eta(P, e, v)}{\bar{v}} .
$$

Hence

$$
\sigma(P) \leqslant \frac{2 n^{2}+(-1)^{n}-1}{4 \underline{v}} \eta(P, e, v) \leqslant \frac{2 n^{2}+(-1)^{n}-1}{8} \mu\left|1-\lambda_{2}(P)\right| .
$$

Corollary 4.2. Let $P$ be a doubly stochastic matrix. Then

$$
\sigma(P) \leqslant \frac{2 n^{2}+(-1)^{n}-1}{8}\left|1-\lambda_{2}(P)\right|
$$

P r o of. If $P$ is reducible, then $\sigma(P)=0$ and (17) holds. If $P$ is irreducible, then it follows from (16) and $\mu=1$.

Corollary 4.3. Let $P$ be an irreducible stochastic matrix and

$$
p=\min \left\{p_{i j}: p_{i j} \neq 0, i \neq j\right\}
$$

Then

$$
\sigma(P) \leqslant \frac{n^{2}}{4 p^{n-1}}\left|1-\lambda_{2}(P)\right|
$$

P r o o f. It follows from $(4.2)$ and $\mu \leqslant(1 / p)^{n-1}$ by [8].

Remark 4.4. Theorem 4.1, Corollary 4.2 and 4.3 partly answer the Hartfiel and Meyer's Conjecture [3]. 


\section{References}

[1] A. Berman and R. J. Plemmons: Nonnegative Matrices in the Mathematical Sciences. Academic Press, New York-San Francisco-London, 1979; SIAM, Philadelphia, 1994.

[2] M. Fiedler: An estimate for the non-stochastic eigenvalues of doubly stochastic matrices. Linear Algebra Appl. 214 (1995), 133-143.

[3] D. J. Hartfiel and C.D. Meyer: On the structure of stochastic matrices with a subordinant eigenvalue near 1. Linear Algebra Appl. 272 (1998), 193-203.

[4] A. W. Marshall and I. Olkin: Inequalities: Theory of Majorization and Applications. Academic Press, New York, 1979.

[5] R. Merris: Laplacian matrices of graphs: a survey. Linear Algebra Appl. 197-198 (1994), 143-176.

[6] R. Merris: A survey of graph laplacians. Linear and Multilinear Algebra 39 (1995), 19-31.

[7] B. Mohar: Laplace eigenvalues of graphs - a survey. Discrete Math. 109 (1992), 171-183.

[8] H. Schneider: Note on the fundamental theorem on irreducible nonnegative matrices. Proc. Edinburgh Math. Soc. 12 (1960/1961), 107-112.

Authors' addresses: X .- D . Z h a n g, Department of Mathematics, Shanghai Jiaotong University, Shanghai 200030 ,Peoples Republic of China, e-mail: xdzhang2@hotmail.com; R. L u o, Department of Mathematics, West Virginia University, Morgantown, WV, 26505, U.S.A., e-mail: luor@math.wvu.edu. 